\title{
Inhaltsverzeichnifs des neunzehnten Bandes, nach den Gegenständen.
}

\section{Reine M a thematik.}

Nr. der

Abbandlung.

1. An aly sis.

2. Theorie der Modular-Functionen und der Modular-Integrale. Von Herrn Prof. Dr. Gudermann zu Münster. (Fortsetzung von No. 1., 10., 15. und 21. im vorigen Bande.) . . . . . . . . . . . . . . . . . . I. 46

8. Fortsetzung dieser Abhandlung. . . . . . . . . . . . . . . II. 119

12. Fortsetzung dieser Abhandlung. . . . . . . . . . . . . . . III. 244

3. Sur la décomposition d'une certaine classe de fonctions. Par Mr. Chr. Jürgensen, de Copenhague. . . . . . . . . . . . . . . . I. 84

5. Ueber die Congruenz $x^{2}+y^{2} \equiv 1$ (mod. $p$ ). (Theorie der trigonometrischen Functionen in Bezug auf Congruenzen.) Von Herrn Theodor Schönemann. II. 93

6. Sur la sommation des transcendentes à différentielles algébriques. Par Mr. Jürgensen, de Copenhague. . . . . . . . . . . . . . . II. 113

7. Démonstration de la formule générale d'intégration indéfinie proposee par Mr. Hill. Par Mr. Ramus, prof. à Copenhague. . . . . . . . . II. 117

11. Theorie der symmetrischen Functionen der Wurzeln einer Gleichung. Allgemeine Sätze uber Congruenzen, nebst einigen Anwendungen derselben. Von Herrn Theodor Schönemann zu Berlin. . . . . . . . III. 231

14. Schlufs dieser Abhandlung. . . . . . . . . . . . . . . . . . IV. 289

13. Note sur l'intégration de l'équation $\frac{d^{n} y}{d x^{n}}=x^{m} \cdot y$ par des intégrales définies. Par E. E. Kummer, Dr. en phil. à Liegnitz. . . . . . . . . . III. 286

16. Ueber die complexen Primzahlen, welche in der Theorie der Reste der $i^{\text {ten }}, 8^{\text {ten }}$ und $12^{\text {ten }}$ Potenzen zu betrachten sind. Von Herrn C. G. J. Jacobi, Professor ordin. an der Universität zu Königsberg in Pr. (Gelesen in der Akademie der Wissenschaften den 16. Mai 18:39.) . . . . . . IV. 314

16. a. Sur le théorême de Wilson. Par Mr. Brennecke, professeur des math. à Berlin. . . . . . . . . . . . . . . . . . . . . . . IV. 319

17. Recherches sur diverses applications de l'Analyse infinitésimale à la T'béorie des nombres. Première partie. Par Mr. G. Lejeune Dirichlet. . . . IV. 324 
10. Beweise einiger geometrischer Lehrsätze. Von Herrn Dr. Bauer in Stettin. III. 205

15. Note von der geodätischen Linie auf einem Ellipsoïd und den verschiedenen Auwendungen einer merkwürdigen analytischen Substitution. Von C. G.J. Jacobi, Professor ordin. zu Königsberg in Pr. (Gelesen in der Königl. Akademie der Wissenschaften zu Berlin am 18. April 1839.) . . . IV. 306

18. Wie sich entscheiden lälst, ob zwei gegebene krumme Flächen auf einander abwickelbar sind oder nicht; nebst Bemerkungen über die Flächen von unveränderlichem Krümmungsmaalse. Von Hrn. Dr. Ferd. Minding zu Berlin.

\section{3. $\mathrm{M}$ e c h a n i k.}

9. Mémoire sur l'équilibre d'un corps solide suspendu à un cordon flexible.

Par M. Pagani, prof. ord. à l'université de Louvain. . . . . . . . III. 185

\section{A nwendung der Mathematik.}

1. Discussion de la forme générale des ondes lumineuses. Par Mr. Plücker, prof. ord. à Bonn. . . . . . . . . . . . . . . . . . . I. 1

4. Note rélatif au précédent mémoire. . . . . . . . . . . . . $\quad$ I. 91 